\title{
INCONTINENCIA URINARIA POST-PROSTATECTOMÍA. EL ESFINTER ARTIFICIAL
}

\author{
Fernando Rodríguez Escobar y Pedro Arañó Bertran.
}

Fundació Puigvert. Unidad de Urología Funcional y Femenina. Barcelona. España.

\begin{abstract}
Resumen.- El esfínter artificial (EA) es actualmente el tratamiento considerado como "gold standard" en el tratamiento de la incontinencia urinaria post prostatectomía radical. La incontinencia de orina post prostatectomía radical es una complicación que se presenta en un $5-25 \%$ de los pacientes sometidos a este tratamiento. Esta complicación genera en los pacientes un deterioro importante en cuanto a su calidad de vida. Tratamientos conservadores y mínimamente invasivos han sido utilizados sin éxito, como por ejemplo los ejercicios de Kegel, el uso de absorbentes, farmacoterapia y electroestimulación o cirugía menor como los inyectables. Esta revisión resume las ventajas y complicaciones del esfinter artificial y su efectividad en el manejo de la incontinencia urinaria tras una prostatectomía radical.
\end{abstract}

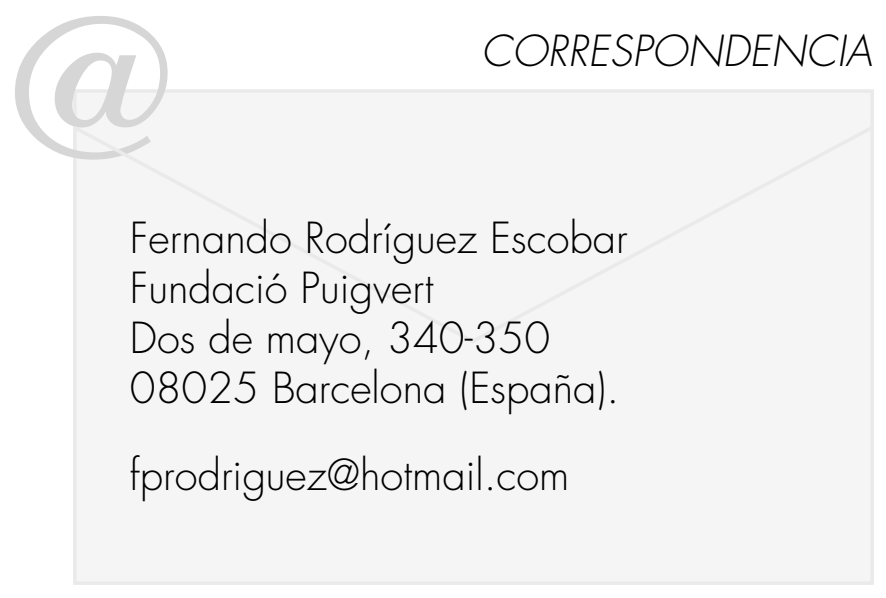

Palabras clave: Esfínter artificial. Incontinencia urinaria. Prostatectomía radical. Cáncer de próstata.

Summary.- Urinary artificial sphincter (UAS) is currenthy the gold standard treatment for urinary incontinence after radical prostatectomy. 5-25\% of the patients undergoing radical prostatectomy develop urinary incontinence. This complication generates important deterioration in the quality of life of these patients. Conservative and minimally invasive treatments, such as Kegel exercises, use of pads, drugs, and electrostimulation, or minor surgery have been used without success. This review summarizes the advantages and complications of the urinary artificial sphincter and its efficacy in the management of urinary incontinence after radical prostatectomy.

Keywords: Urinary artificial sphincter. Urinary incontinence. Radical prostatectomy. Prostate cancer.

\section{INTRODUCCIÓN}

La incontinencia urinaria tras una prostatectomía radical por patología maligna es una complicación bien conocida y temida debido al impacto tan importante que tiene sobre la calidad de vida de los pacientes.

La evaluación de estos pacientes es similar a la que se realiza para otros tipos de incontinencia e incluye una buena historia clínica, diario miccional, examen físico y estudio urodinámico.

La incidencia de incontinencia después de prostatectomía radical ha sido fuente de controversia en los últimos años debido a que las tasas registradas variaron significativamente. Dicha incidencia probablemente disminuyó a partir de los años 90 , por los avances en la técnica quirúrgica y la detección más 
temprana de la enfermedad en sus primeros estadios en pacientes más jóvenes. Sin embargo, la prevalencia de la incontinencia ha aumentado debido al incremento en el número de procedimientos efectuados cada año.

Aproximadamente entre el $5-25 \%$ de los pacientes sometidos a una prostatectomía radical experimentaran incontinencia, con un $70-90 \%$ de pacientes secos y sin necesidad de utilizar protección un año despues de la cirugía, y $80-95 \%$ sin pérdidas nocturnas. La razón del amplio rango en la incidencia de la incontinencia es el uso de diferentes definiciones de continencia y los métodos para cuantificarla. Algunas series recientes utilizan definiciones que incluyen "control total", "goteo ocasional pero sin compresa", y "menos de una compresa".

El esfínter artificial ha ofrecido resultados satisfactorios e impacto positivo sobre la calidad de vida de los pacientes. La incontinencia después de la prostatectomía radical se asocia con una disminución de la satisfacción sexual, una limitación de actividades cotidianas y con un deseo de someterse nuevamente a una cirugía (1-4). Los mayores grados de pérdida urinaria se correlacionan con más "molestias" y cambios significativos en la calidad de vida $(5,6)$.

\section{Desarrollo (historia) del esfínter artificial}

Como la incontinencia posprostatectomía es causada la mayoría de las veces por daño del esfínter, muchos urólogos han trabajado en la idea de un dispositivo que mejore la insuficiencia esfinteriana. En 1947, Foley fue el primero en describir un esfínter artificial que se asemejaba a un "sphygmomanometer" que consistía en un manguito inflable periuretral conectado a una bomba desmontable que llevaba el paciente en su bolsillo (7). Como era un dispositivo externo y hecho de un material NO inerte, su éxito fue mínimo y su popularidad disminuyó rápidamente. En Junio de 1972, Scott implantó su nuevo dispositivo en una mujer de 36 años con mielodisplasia e inicio la era moderna del implante de los esfínteres artificiales (8).

Sin embargo, no fue hasta 1974 cuando Scott et al. presentan su serie inicial de 34 pacientes con un porcentaje de éxito de $79 \%$ y con mayor durabilidad en pacientes tras una prostatectomía radical, lo que captó la atención de los fabricantes. American Medical Systems (AMS, Minnetoka, Minnesota) fue la primera en patentar este esfínter, el modelo AS $721^{\mathrm{TM}}$. Este modelo despues fue asociado con un alto porcentaje de fallos mecánicos y además era muy incómodo de implantar. Durante la siguiente década el AS $721^{\text {TM }}$ fue modificado y simplificado, estas mejoras aumentaron la fiabilidad y disminuyeron el porcentaje de erosiones uretrales, infecciones, fallo de la válvula, etc. (9).

El primer paso de este proceso fue el desarrollo del AMS 742 ${ }^{\mathrm{TM}}$, el cual tenía una sola bomba, manguito y balón presurizador. Este, fue la primera prótesis con manguito de cerrado automático, de manera que la alta presión dentro del balón llenaría automáticamente el manguito.

En 1983, se lanzó el AMS 800 ${ }^{\mathrm{TM}}$, el cual tenía incorporado un botón de desactivación dentro del sistema. Desde entonces, se han realizado algunas mejoras al modelo AMS $800^{\mathrm{TM}}$, incluyendo un revestimiento de silicona al manguito con la finalidad de disminuir el riesgo de daño a este nivel. Actualmente, el modelo AMS $800^{T M}$ es el dispositivo más estudiado y más comúnmente utilizado en pacientes con incontinencia urinaria post-prostatectomía.

\section{Factores de riesgo}

\section{Fisiología de la continencia}

Para entender la incontinencia post-prostatectomía es importante repasar primero la anatomía y la fisiología normal de la continencia en el hombre, antes y después de la prostatectomía. La continencia normal en el hombre requiere un detrusor estable $y$ distensible, y una uretra competente. Cada una de estas estructuras anatómicamente diferentes a su vez requiere una innervación normal, musculatura lisa y estriada normal y estructuras de sostén y componentes del tejido blando intactos. En condiciones normales, la vejiga debe tolerar cantidades de orina a baja presión sin presentar contracciones involuntarias. Un esfínter que funcione bien debería ser capaz de resistir aumentos de la presión abdominal y permanecer cerrado y luego relajarse de manera apropiada durante la micción voluntaria.

El mecanismo normal del esfínter urinario en el hombre puede dividirse en dos unidades separadas desde el punto de vista funcional: el esfínter uretral proximal y el distal. El primero esta conformado por el cuello vesical, la próstata y la uretra prostática. Está inervado por fibras autónomas simpáticas del nervio hipogástrico. Es precisamente a este nivel que se ve afectada la continencia luego de una prostatectomía radical.

La uretra distal se extiende desde el verumontanum hasta el bulbo y esta compuesto por: el rabdoesfinter, musculatura esquelética parauretral extrínseca y las aponeurosis. 


\section{Fisiopatología de la incontinencia}

La incontinencia aparece como resultado de una disfunción vesical, una disfunción esfinteriana o la combinación de ambas.

Estudios urodinámicos prequirúrgicos en hombres que fueron sometidos a una prostatectomía radical mostraron una incidencia de hiperactividad del detrusor del 17 al 32\% (10-12).

El mecanismo esfinteriano proximal se elimina durante la prostatectomía radical. El mal funcionamiento del esfínter distal puede deberse a una lesión directa de este o de las estructuras de sostén, una lesión de su inervación o a una lesión preexistente. Esta disfunción se debe a la incapacidad del esfínter para resistir incrementos de presión abdominal y puede manifestase con síntomas de incontinencia de esfuerzo, postural o total.

\section{Factores de riesgo}

Los factores de riesgo de incontinencia posterior a la prostatectomía radical son: la edad del paciente en el momento de la cirugía, estadio de la enfermedad, técnica quirúrgica, la experiencia del cirujano, diagnóstico prequirúrgico de detrusor hiperactivo, radioterapia previa o antecedente de RTUP.

Existe la evidencia de que en el paciente anciano se observa una atrofia del rabdoesfínter (14) y degeneración nerviosa (15), lo que se traduce en un mayor riesgo de incontinencia postprostatectomía en estos pacientes.

En la mayoría de las series no se detectó relación entre el estadio de la enfermedad y las tasas de incontinencia $(5,17,18)$. Sin embargo, en ciertos casos el estadio de la enfermedad podría afectar la técnica quirúrgica (Ej.: la conservación de nervios) y las tasas podrían ser mayores, pero parece ser un reflejo de la técnica quirúrgica y no del estadio de la enfermedad (17).

Con respecto a la técnica quirúrgica, los parámetros implicados en la continencia podrían explicar lo difícil que resulta entender los beneficios de ciertos detalles técnicos. Entre estos detalles estarían: la conservación de los paquetes neurovasculares, la preservación y reconstrucción del cuello vesical y la conservación de los ligamentos puboprostáticos.

No queda claro si la preservación de los paquetes nerviosos contribuye con la función del esfínter externo (12), o si la disección mas cuidadosa alrededor del esfínter, necesaria para la conservación de las bandeletas, es la responsable de la mejor continencia.

En cuanto a la preservación de los ligamentos puboprostáticos, Lowe (1997) mostró una tasa de continencia del $100 \%$ con esta técnica comparada con el 84 al $89 \%$ de otras (13). También informó mayor rapidez hasta la continencia total, con una media de 1,6 meses, y llegó a la conclusión de que el mantenimiento de la fijación uretral membranosa al pubis era importante para la función esfinteriana normal.

Hay estudios que informan sobre una mejoría de la continencia a los tres meses postprostatectomía en aquellos pacientes donde se realizó la preservación del cuello vesical (14), pero no se observaron cambios a los 6 y 12 meses (8).

Los pacientes sometidos a radioterapia previa tienen un riesgo más alto de desarrollar incontinencia después de la prostatectomía radical. Las tasas de incontinencia severa tras prostatectomía post radioterapia varían de un $57 \%$ hasta $64 \%$.

\section{Evaluación de la incontinencia postprosta- tectomía}

La importancia o gravedad de la incontinencia postprostatectomía se pueden determinar por su impacto sobre la calidad de vida del paciente y las posibilidades terapéuticas que se le puedan ofrecer.

La mayoría de los autores están de acuerdo con que se necesita un período de seguimiento postquirúrgico de por lo menos 12 meses antes de determinar el grado final de incontinencia.

La evaluación del paciente comienza con una anamnesis completa y detallada. Aspectos importantes son una descripción del tipo y la severidad de la incontinencia así como sus desencadenantes. La severidad puede determinarse por la cantidad de episodios por día, la necesidad y cantidad de protección, y el impacto de la incontinencia sobre su calidad de vida. Debería determinarse la presencia de otros síntomas del tracto urinario inferior como urgencia, polaquiuria o disminución del flujo urinario.

El escape como síntoma de la incontinencia de esfuerzo predice en un porcentaje elevado de pacientes una disfunción esfinteriana. La incontinencia de urgencia como síntoma de una disfunción vesical no parece ser tan valiosa y su diagnóstico no puede realizarse sin la ayuda de un estudio urodinámico 
completo, también es importante conocer el estado del paciente antes de la cirugía en relación a los síntomas del tracto urinario inferior.

\section{Estudio urodinámico}

EI EUD es la única manera de realizar un diagnóstico preciso de la etiología de la incontinencia. El estudio es importante no solo para determinar la causa de la incontinencia sino también para valorar el tipo de tratamiento a efectuar. El estudio tiene como objetivos principales el de confirmar o no la existencia de una disfunción vesical o esfinteriana durante la fase de llenado y establecer si hay obstrucción durante la micción.

Durante el llenado, la maniobra de Valsalva y la tos se efectúan a intervalos regulares para demostrar la incontinencia de esfuerzo. La disfunción esfinteriana se define como la demostración de pérdida de orina con incremento de la presión abdominal, en ausencia de elevación en la presión del detrusor.

\section{Tratamiento}

El tratamiento de la incontinencia postprostatectomía varía entre conservador y agresivo, y debe adaptarse al individuo en función de su causa, grado, efecto sobre la calidad de vida y expectativa frente al tratamiento. Algunos pacientes con incontinencia importante estarán satisfechos con algunos cambios en el estilo de vida y no requerirán ningún tratamiento, mientras que otros con solo grados leves de incontinencia pueden demandar tratamientos agresivos.

Es importante considerar las causas de disfunción vesical y esfinteriana en la planificación del tratamiento. En los casos de disfunción vesical o esfinteriana puras el tratamiento suele ser evidente, pero en los casos de incontinencia mixta no son tan simples. Algunos autores consideran que la disfunción vesical debería tratarse antes de tratar la insuficiencia esfinteriana.

\section{El esfinter artificial}

Una variedad de dispositivos han sido diseñados con la idea de tratar la insuficiencia esfinteriana en el varón desde mediados del siglo 18 (14). Desde entonces se han fabricado elementos tanto de uso externo como de implante interno. Hoy en día es considerado como "gold standard" el esfínter artificial diseñado por F.B. Scott, W.E. Bradley, y G.W.
Timm en 1973 (8). El diseño original sufrió numerosas modificaciones, pero el principio básico ha sido siempre el mismo. Consiste en un sistema hidráulico con un manguito que rodea la uretra, un balón que regula la presión del sistema y una bomba de activación que se coloca en el escroto.

\section{Indicaciones}

La indicación para el implante de un esfínter artificial es la incontinencia urinaria severa, incontinencia que afectará la calidad de vida del paciente. En la gran mayoría de pacientes varones la incontinencia es secundaria a una prostatectomía radical, la vejiga neurógena es la segunda causa más frecuente. La radioterapia previa a nivel de la pelvis no es una contraindicación (15).

Estudios recientes informan sobre resultados similares en pacientes irradiados y no irradiados, aunque una alta incidencia de atrofia uretral, erosión e infección que han requerido de revisión quirúrgica ha sido reportada en los pacientes irradiados en comparación con aquellos que no lo han sido $141 \%$ vs. $11 \%$ ). Obviando esta observación, la continencia a largo plazo y la satisfacción de los pacientes aparentemente no se ve afectada por la radioterapia (15). Los pacientes con problemas de contractibilidad del detrusor que necesitan realizar maniobra de Valsalva para orinar o aquellos pacientes neurógenos con detrusor acontráctil no parecen tener un riesgo sobreañadido de complicaciones. Vale la pena resaltar el hecho de que aquellos pacientes a los que se las ha realizado algún procedimiento antiincontinencia previo tienen una posibilidad mayor de complicaciones luego de la colocación del esfínter artificial (16).

\section{Técnica quirúrgica}

El manguito del esfínter es implantado alrededor de la uretra bulbar a través de una incisión en la línea media del periné, mientras que tanto el balón reservorio como la válvula de activación/desactivación son insertadas a través de una incisión suprapúbica.

\section{Implante de manguito oclusivo en la uretra bulbar}

A través del abordaje perineal (Figura 1), previa colocación de sonda vesical, se realiza la localización y disección de la uretra bulbar respetando en la medida de lo posible el músculo bulbocavernoso. Se diseca la uretra al nivel donde decidimos colocar el manguito oclusivo hasta que se pueda pasar con un disector el medidor de forma amplia (Figura 2). 
A continuación, una vez colocado el medidor se medirá el grosor de la uretra y por ende la longitud del manguito. Colocación del manguito uretral. El tubo conector de este elemento se pasara vía subcutánea hasta salir a nivel de la incisión suprapubica utilizando para esto una aguja pasa tubos que se encuentra entre los componentes del sistema (Figura 3).

Luego se coloca el balón reservorio en un espacio paravesical, para esto se incide mínimamente sobre el músculo y la fascia a través de la incisión suprapúbica, una vez colocado el balón se llenara con 22-23cc de la solución de contraste o suero fisiológico. La bomba se coloca en la bolsa escrotal (Figura 4).

Para finalizar se realizan las conexiones entre los tres elementos y se cierran las incisiones. Es importante asegurarse de que el sistema quede desactivado. La sonda uretral será retirada a las 24 horas de la cirugía.

Desde hace unos años algunos grupos vienen utilizando un abordaje único, en el que se realiza una incisión escrotal a través de la cual se colocaran los tres elementos del sistema, el manguito, la bomba en la bolsa escrotal, y el reservorio debajo de la fascia transversalis (17). Esta vía de abordaje, según lo describen sus autores, podría ser más fácil y rápida una vez dominada. Sin embargo debemos ser cuidadosos antes de considerar esta vía como la técnica de elección a la hora de colocar un esfínter artificial ya que el implante del manguito en la porción proximal de la uretra bulbar parece ser más dificultosa, y actualmente no se cuenta con resultados de esta técnica a largo plazo.

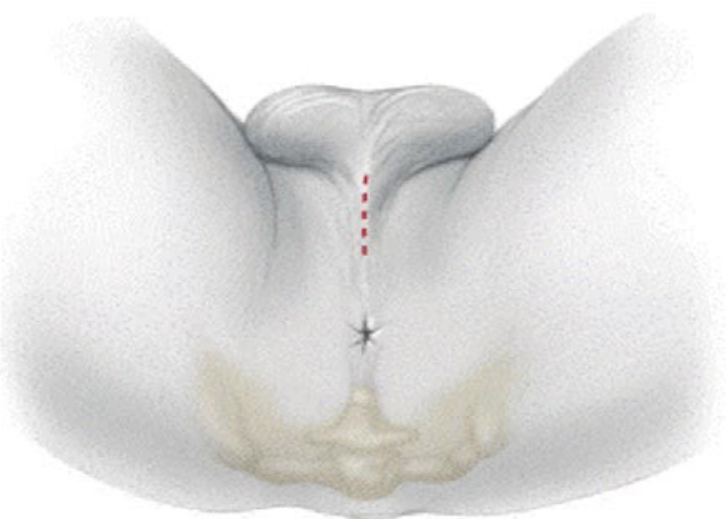

FIGURA 1. Incisión línea media perineal para acceder a la utretra bulbar.

\section{Postoperatorio}

Al día siguiente de la colocación del esfínter artificial con contraste se debe realizar una radiografía simple de abdomen que incluye toda la pelvis para comprobar la correcta localización de todos los elementos del sistema y su desactivación. También se recomienda una pauta antibiótica vía oral durante al menos una semana y la utilización de un suspensorio que ayude a prevenir los hematomas escrotales.

A los dos meses de la colocación del esfínter artificial se realizará activación del mismo y se enseñará al paciente su correcta manipulación, se repetirá la radiografía simple para verificar la activación del sistema en caso de haber utilizado contraste.

\section{Complicaciones}

Las complicaciones de la colocación del esfínter artificial pueden ser clasificadas en mecánicas y no mecánicas. Las no mecánicas incluyen infección, erosión y atrofia uretral, y las mecánicas podrían ser el resultado de un escape de la solución de contraste de los tubos conectores o del reservorio, o mal funcionamiento de la válvula.

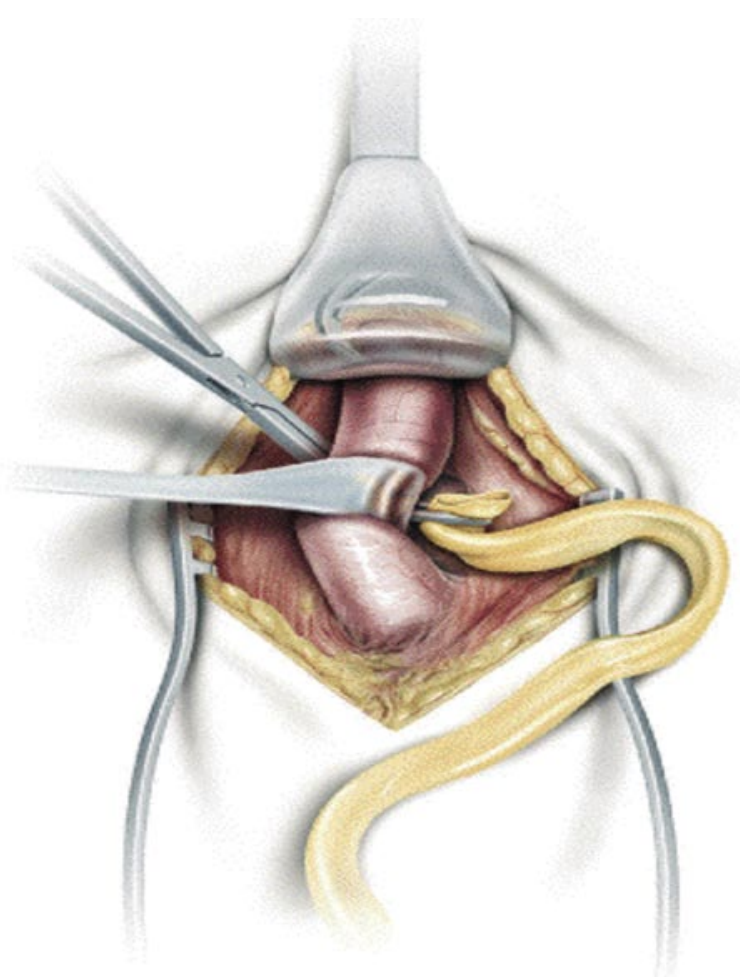

FIGURA 2. Paso del disector para la colocación del medidor uretral. 
El porcentaje de infección está alrededor del $1.8-10 \%(3.4 \%)(15)$, con los valores más altos en pacientes irradiados. La infección es frecuentemente causada por Staphylococcus epidermidis. Si el proceso infeccioso aparece durante el postoperatorio inmediato, se sospecha que pueda haber sido originado por contaminación de la prótesis en el momento del implante. La infección más tardía podría ser causada por vía hematógena procedente de otro foco. La mayoría de las prótesis infectadas no podrán salvarse con tratamiento antibiótico y tendrán que ser extraídas. La colocación de un nuevo esfínter artificial se podrá realizar tras un período de entre 3 y 6 meses una vez resuelto el proceso infeccioso, ésta se tendrá que colocar a otro nivel uretral o bien valorar la posibilidad de la colocación a través de los cuerpos cavernosos.

El porcentaje de erosiones ha disminuido significativamente (1-3\%) luego de establecer que el dispositivo se debe activar por lo menos 2 meses después de ser colocado (18). La erosión del esfínter artificial puede ser precipitada por infección, disminución de la vascularización por radioterapia previa, presión excesiva a nivel del manguito uretral, manguito uretral de un tamaño menor al indicado por

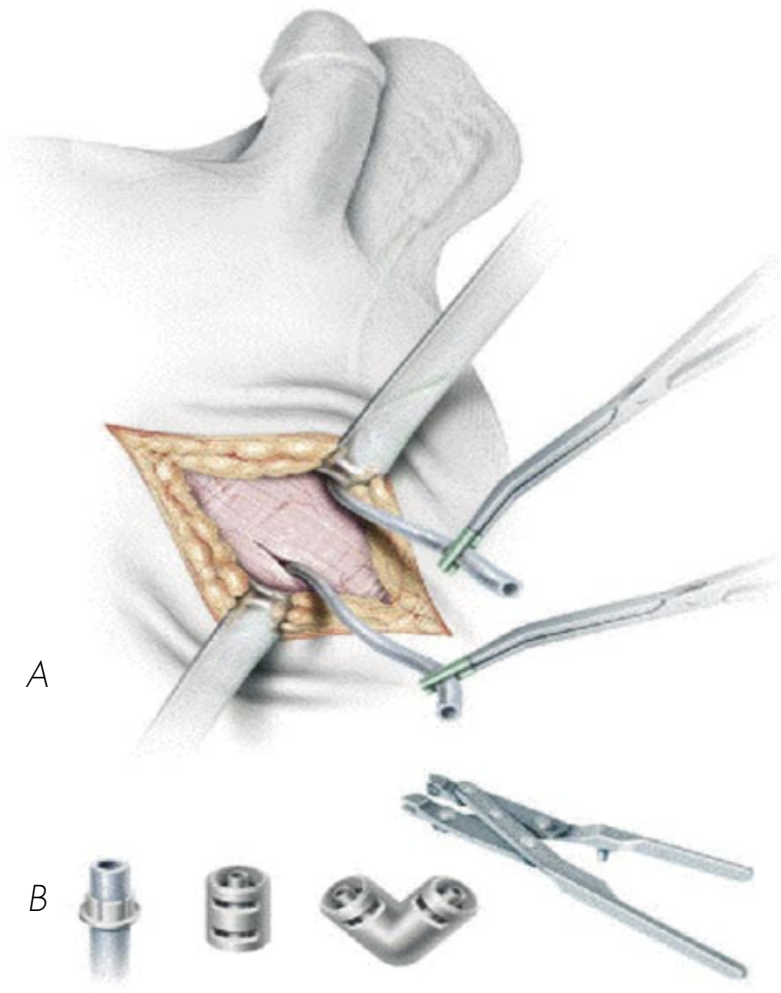

FIGURA 3. Incisión suprapúbica para la exposición de los tubos conectores. el medidor, sondaje traumático. Es muy importante desactivar el sistema si la uretra tiene que ser manipulada (sondaje, cistoscopia, etc.).

La atrofia uretral es una causa común de la recurrencia de la incontinencia, con una incidencia del 3-9\%. Dentro de las opciones terapéuticas de esta complicación se encuentra la colocación de un segundo manguito a nivel más proximal (19) o de uno más pequeño.

La pérdida de solución de contraste por parte del sistema no es infrecuente y puede ser el resultado de perforaciones o pequeñas fracturas a nivel de los tubos conectores, manguito o reservorio. El mal funcionamiento de la válvula es frecuentemente causado por problemas a nivel de los tubos. Esta es una complicación que se puede prevenir asegurándose el correcto "purgado" de todos los elementos, evitando el acodamiento de los tubos por un tamaño redundante, y educando al paciente para un correcto manejo del esfínter.

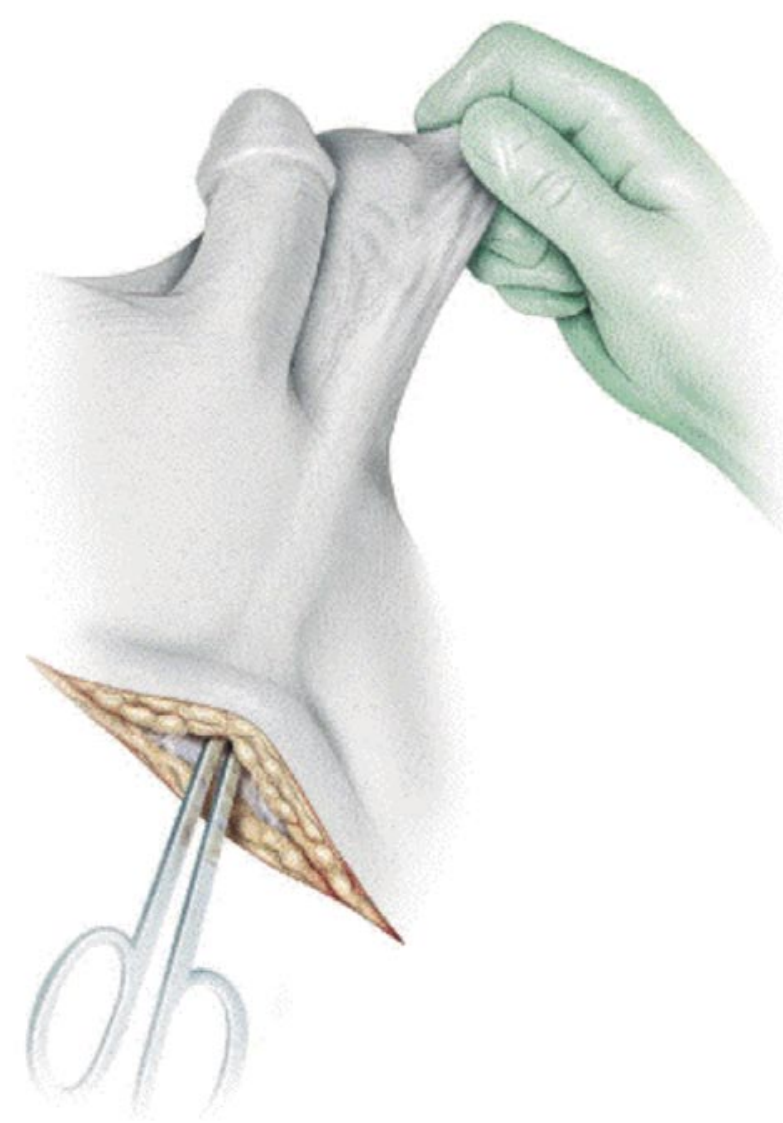

FIGURA 4. Colocación de la válvula a nivel escrotal. 
La persistencia de la incontinencia de esfuerzo puede ocurrir en más de un $15 \%$ de los pacientes tras la colocación del esfínter artificial. Una vez descartadas las causas expuestas anteriormente, se podría pensar en la presencia de un manguito uretral de un diámetro mayor al requerido o al implante de un reservorio de muy poca presión. El manejo de estas complicaciones comienza con el cambio del manguito por uno de tamaño correcto. También existe la posibilidad de recolocar el manguito más proximal o incluso de colocar un segundo manguito (19).

\section{Calidad de vida}

Litwiller et al. (18) estudiaron la satisfacción a largo plazo de los pacientes tratados con el implante de un esfínter artificial por incontinencia de orina postprostatectomía radical. En este estudio, la continencia fue definida como la ausencia de escape de orina. El período de seguimiento fue de 23.4 meses (3-1 14) en 50 pacientes. Los autores concluyeron que el nivel de satisfacción de los pacientes fue uniformemente alto $(90 \%)$ y esto no fue dependiente de una completa continencia sino de una mejora significativa de las pérdidas. Observaron que aunque persistieran las pérdidas, 23 de $25(92 \%)$ de los pacientes recomendarían el esfínter artificial a un amigo con el mismo problema de incontinencia.

La elevada incidencia de revisión quirúrgica posterior a la colocación de la prótesis observada en aquellos paciente con antecedentes de radioterapia externa o braquiterapia, no afecta la satisfacción ni la calidad de vida de estos individuos, obteniendo resultados similares a aquellos que no han sido irradiados (20).

\section{BIBLIOGRAFIA y LECTURAS RECOMENDADAS ( ${ }^{*}$ lectura de interés $y^{* *}$ lectura fundamental)}

1. Fowler FJ, Barry MJ, Lu-Yao G, et al. Patient-reported complications and follow-up treatment after radical prostatectomy: The national Medicare experience: 1988-1990 (update June 1993). Urology, 1993; 42:622-9.

2. Herr H. Quality of life of incontinent men after radical prostatectomy. J Urol, 1994; 151:652-4.

3. McCammon KA, Klom P, Main B, et al. Comparative quality of life analysis after radical prostatectomy or external beam radiation for localized prostate cancer. Urology, 1999; 54:509-16.

4. Kao TC, Cruess DF, Garner D, et al. Multicenter patient self-reporting questionnaire on impotence, incontinence and stricture after radical prostatectomy. J Urol, 2000; 163:858-64.
5. Jonler M, Messing EM, Rhodes PR, et al. Sequelae of radical prostatectomy. Br J Urol, 1994:74:352-8.

6. Donnellan SM, Duncan HJ, MacGregor RJ, et al. Prospective assessment of incontinence after radical retropubic prostatectomy: Objective and subjective analysis. Urology, 1997; 49:225-30.

7. Foley FEB. Artificial sphincter new device and opetation for control of enuresis and urinary incontinence. J Urol, 1974; 58:250-9.

8. Scott FB, Bradley WE, Timm GW. Treatment of urinary incontinence by implantable prosthetic sphincter. Urology, 1973; 1:252-9.

9. Scott FB, Bradley WE, Timm GW. Treatment of urinary incontinence by implantable prosthetic sphincter. J Urol, 1974; 112: 75-80.

10. Abseil SR, Knotty B, Schmidt RA, et al. Preoperative urodynamic evaluation: Does it predict the degree of urinary continence after radical, retropúbic prostatectomy? Urol Int, 1994; 53:68-73.

11. Hammerer P, Hulland H. Urodynamic evaluation of change in urinary control after radical retropubic prostatectomy. J Urol, 1997; 157:233-6.

12. Kleinhans B, Gerharz E, Melekos M, et al. Changes of urodynamic findings after radical retropubic prostatectomy. Eur Urol, 1999; 35:217-22.

13. Tomaschi W, Suster G, Holtl W. Bladder neck strictures after radical retropubic prostatectomy: Still an unsolved problem. Br J Urol, 1998; 81: 823-6.

14. Brunett AL, Mostwin JL. In situ anatomical study of the male urethral sphincteric complex: Relevance to continence preservation following major pelvic surgery. J Urol, 1998; 160:1301-6.

15. Chao R, Mayo ME: Incontinence after radical prostatectomy: Detrusor or sphincteric causes. J Urol, 1995; 154:16-8.

16. Meulen PH, Zambon V, Kessels AG, van Kerrebroeck PE. Quality of life, functional outcome and durability of the AMS 800 artificial urinary sphincter in patients with intrinsic sphincter deficiency. Urol, 2003; 71:55-60.

**17. Wilson SK, Delk JR, 2nd, Henry GD, Siegel AL. New surgical technique for sphincter urinary control system using upper transverse scrotal incision. J Urol, 2003; 169:261-4.

18. Litwiller SE, Kim KB, Fone PD, White RW, Stone AR. Post-prostatectomy incontinence and the artificial urinary sphincter: along-term study of patient satisfaction and criteria for success. J Urol, 1996; 156:1975-80.

*19. Saffarian A, Walsh K, Walsh IK, Stone AR. Uretrhal atrophy after artificial urinary sphincter placement: is cuff downsizing effective? J Urol, 2003; 169:567-9.

20. Gomha MA, Boone TB. Artificial urinary sphincter for post-prostatectomy incontinence in men who prior radiotherapy a risk and outcomes analysis. J Urol, 2002; 167:591-6. 